\title{
IDENTIFICAÇÃO E RASTREAMENTO DE VEÍCULOS UTILIZANDO FLUXO ÓPTICO
}

\section{IDENTIFYING AND TRACKING VEHICLES BY USING THE OPTICAL FLOW}

Fernando Souza Castro ${ }^{1}$, Francisco Assis da Silva ${ }^{1}$, Mário Augusto Pazoti $^{1}$, Danillo Roberto Pereira ${ }^{1}$, Leandro Luiz de Almeida ${ }^{1}$, Almir Olivette Artero ${ }^{2}$

\footnotetext{
${ }^{1}$ Faculdade de Informática - FIPP, Universidade do Oeste Paulista - UNOESTE e-mail: fafer_castro@hotmail.com, \{chico, mario, danilopereria, Ilalmeida\}@unoeste.br ${ }^{2}$ Faculdade de Ciências e Tecnologia - FCT, Universidade Estadual Paulista - Unesp e-mail: almir@fct.unesp.br
}

RESUMO - Controlar o tráfego de veículos é uma tarefa muito difícil de ser realizada. Essa atividade representa um grande desafio na busca por soluções que possam ajudar o controle e a fiscalização de tráfego de veículos em vias terrestres. Neste trabalho considera-se o emprego do método diferencial, proposto por Lucas e Kanade, para estimação do Fluxo Óptico, como ferramenta computacional para rastrear e classificar veículos a partir de frames de imagens de vídeo. Os veículos encontrados em cada frame são classificados conforme o seu tamanho: pequeno, médio e grande porte. Os resultados deste trabalho podem ser aplicados, por exemplo, em sistemas de contagem de veículos em rodovias. Outra aplicação desse trabalho, que é de grande valia seria para a construção de semáforos inteligentes, que controlam os tempos de bloqueio e liberação da via baseados no fluxo de veículos.

Palavras Chaves: Fluxo Óptico; Rastreamento de Veículos; Contagem de Veículos; Semáforo Inteligente.

ABSTRACT - Controlling the traffic of vehicles is a very hard task to be realized. It is represents a challenge in the search for solutions that can help control and vehicle traffic monitoring on roads. In this work we consider the use of the differential method, proposed by Lucas and Kanade, to estimate the optical flow, such as computational tool to track and classify vehicles from video frame by frame. The vehicles are found in each frame are classified by its size: small, medium and large. The results of this work can be applied, for example, in systems of count of vehicles in highways. Another use for this work, which is a high value, would be to construct intelligent traffic lights, which control the block and freedom of the pathway based on the flow of

Recebido em: 04/04/2015 Revisado em: 29/04/2015 Aprovado em: 06/06/2015 vehicles.

Key words: Optical Flow; Vehicle Tracking; Counting of Vehicles; Intelligent Traffic Lights. 


\section{INTRODUÇÃO}

Com o grande aumento da frota de veículos nas vias das cidades e rodovias, o controle viário tem se mostrado cada vez mais complexo. Um conjunto de semáforos que não utiliza nenhum tipo de controle para interromper ou liberar a passagem dos veículos e somente usa o tempo préestabelecido, pode acarretar grandes engarrafamentos. Estes dificultam em muitas vezes, por exemplo, a circulação de viaturas de saúde e segurança, devido ao fato de não conseguirem trafegar e atender aos chamados da população. A contagem de veículos em uma rodovia não pedagiada também apresenta uma dificuldade, quando feita manualmente (BRITO, 2011).

Segundo Chen, Lin e Chen (2007), os sistemas de vigilância de tráfego precisam ser discutidos e estudados, pois podem fornecer informações significativas e úteis, tais como excesso de velocidade e violação no trânsito. Ao analisar esses problemas pode-se reduzir congestionamentos e melhorar a mobilidade.

Segundo Tannús, Soares e Costa (2013) o trânsito hoje é um caos na maioria das cidades ao redor do mundo. O problema encontra-se principalmente nos engarrafamentos, que trazem problemas para o deslocamento de usuários, aumento da poluição e aumento de acidentes causando um grande impacto na qualidade de vida da população e na economia dos países, ocasionando tempo, esforço e gastos desnecessários.

$$
\text { Uma das soluções para ajudar a }
$$
resolver os problemas encontrados no trânsito é utilizar recursos da área de Visão Computacional para identificar e rastrear veículos. Isso pode ser aplicado no desenvolvimento de semáforos inteligentes, em que um conjunto de semáforos é gerenciado por um sistema computacional para interromper ou liberar a passagem de veículos, conforme as necessidades encontradas em cada momento. Em um horário de pico, por exemplo, uma das vias que pertence a um semáforo pode apresentar um maior fluxo de veículos ou algum veículo estar causando engarrafamento em uma determinada via. Pelo uso de câmeras de vídeo, o sistema computacional poderia contar a quantidade de carros e aumentar o tempo de abertura do semáforo para aquela via, possibilitando a diminuição do engarrafamento. Poderia também ser aplicado em rodovias para contar a quantidade de veículos que passam em um determinado trecho, identificando-os por tipo (pequeno, médio e grande porte) com o uso de câmeras.

Sobral (2012) afirma que o rastreamento de objetos envolve o problema de estimar a trajetória de um determinado objeto (ou múltiplos objetos) em uma 
sequência de frames de um vídeo, ou seja, o algoritmo utilizado para o rastreamento precisa garantir que 0 objeto tenha identidade única ao longo do vídeo. O rastreamento é fundamental para estimar o comportamento do objeto analisando sua trajetória.

Ao rastrear um objeto, muitas vezes é necessária uma representação ou modelo que descreva a forma, aparência ou características que o definem. Com isso, espera-se que o recurso de Fluxo Óptico seja mais adequado para o rastreamento de veículos, em que é feita uma comparação entre o frame anterior e o frame atual, mapeados em um vetor de deslocamento, podendo assim analisar o deslocamento do objeto (SOBRAL, 2012).

Este trabalho apresenta uma metodologia para classificar e rastrear veículos, utilizando apenas recursos de Visão Computacional com o uso de câmera de vídeo, sem o uso de outros tipos de sensores.

Um sensor bastante utilizado para quantificar os veículos que trafegam pelas vias é chamado de sensores de eixo, que são um par de tubos de borracha estendidos de forma perpendicular ao fluxo de trânsito, e dentro destes tubos existem válvulas pneumáticas que são acionadas quando os veículos passam por elas.

Para o desenvolvimento deste trabalho, foi utilizada a técnica de Fluxo
Óptico de Lucas e Kanade (1981) para classificar e rastrear veículos como: pequeno, médio e grande porte, sendo assim possível quantificar os veículos nestas classes.

As demais seções deste trabalho estão organizadas da seguinte maneira: na Seção 2 são descritos os trabalhos relacionados a este; na Seção 3 são apresentados os fundamentos teóricos; na Seção 4 é exposta a metodologia proposta; a Seção 5 descreve os experimentos realizados; na Seção 6 são apresentados os resultados obtidos; a na Seção 7 encontram-se as considerações finais do trabalho.

\section{TRABALHOS RELACIONADOS}

Tannús, Soares e Costa (2013) desenvolveram um algoritmo com 0 propósito de contar os veículos que trafegam nas vias. Para segmentar as imagens utilizaram o modelo de misturas gaussianas, com o objetivo de dividir a imagem em dois tipos de regiões: cenário e objetos móveis. A contagem dos objetos no cenário é realizada através do foreground (imagem dos objetos obtidos na segmentação das imagens). Uma linha horizontal é posicionada na metade do frame e quando o objeto cruza essa linha os pixels do objeto são identificados. Os resultados alcançados por Tannús, Soares e Costa (2013) podem ser analisados a partir da Tabela 1, em que a coluna "Caso" 
apresenta o nome do arquivo, a coluna "Qm" a quantidade de veículos obtida manualmente, a coluna "Qa" a quantidade de veículos encontrados pelo algoritmo, a coluna "D(s)" o tempo de duração do vídeo em segundos e a coluna " $P(s)$ " o tempo gasto para processar o vídeo em segundos.

Tabela 1. Resultados obtidos.

\begin{tabular}{l|c|c|c|c}
\hline Caso & Qm & Qa & D(s) & P(s) \\
\hline t001.avi & 32 & 32 & 148 & 300 \\
\hline t002.avi & 16 & 16 & 56 & 114 \\
\hline t003.avi & 75 & 75 & 300 & 600 \\
\hline t004.avi & 19 & 19 & 81 & 164 \\
\hline t005.avi & 5 & 5 & 20 & 44 \\
\hline
\end{tabular}

Fonte: (Tannús, Soares e Costa, 2013).

Brito (2011) realizou um trabalho com o objetivo de descrever frame a frame a trajetória dos veículos e gerar um relatório em arquivo texto. $\mathrm{O}$ primeiro passo foi obter a imagem de fundo através de um trecho do vídeo. Para obter a imagem de fundo foi realizado o cálculo da mediana da intensidade das cores em cada pixel, e ordenado por ordem crescente em três vetores, em que cada vetor representa os canais RGB (Red, Green, Blue), a mediana de cada vetor compõe a cor de fundo do pixel analisado, esse processo foi realizado em todos os pixels da imagem. Com a imagem de fundo foi realizada a subtração do fundo com o frame atual, obtendo-se somente os veículos que se encontram na imagem.
Para identificar os veículos, templates (imagens que se aproximam ao tamanho e a forma dos veículos) foram sobrepostos a eles para identificar a região de cada veículo na imagem.

Para realizar o rastreamento dos pontos foi utilizado a técnica de Lucas e Kanade (1981) disponível na biblioteca OpenCV. Segundo o autor, através dos processos de templates e Fluxo Óptico foi possível acompanhar toda trajetória dos veículos. Os resultados esperados pelo autor foram parcialmente alcançados, pois o algoritmo necessita que uma imagem de fundo seja anteriormente carregada em memória. No processo em que se utiliza templates, os veículos como caminhões foram ignorados devido às inúmeras formas e tamanhos.

Sobral (2012) realizou um trabalho com a proposta baseada em um contexto global, que seja possível classificar o estado do trânsito como baixo, médio e alto congestionamento. $\mathrm{O}$ primeiro passo foi definir uma região de interesse no vídeo escolhendo uma área que apresente a maior concentração de movimento. Para segmentar o congestionamento dos veículos, foi utilizado à técnica de subtração de fundo, por ser uma técnica de uso comum para segmentar objetos que estejam em movimento. Após o processo de subtração de fundo foi realizado a estimação da densidade 
de veículos, adquirida pela contagem dos pixels não nulos da máscara de foreground obtida na subtração de fundo, e para o rastreamento da densidade de veículos foi utilizado o método de Lucas e Kanade (1981). Para estimar a velocidade do aglomerado de veículos foi realizado o deslocamento médio dos pontos de interesse por frame. O autor cita alguns critérios e limitações do sistema, como a necessidade do uso de câmeras estáticas, utilizar apenas uma via de trânsito e não utilizar o sistema em ambientes noturnos.

\section{REFERENCIAL TEÓRICO}

Nessa seção são apresentados os fundamentos teóricos a respeito de Fluxo Óptico.

\subsection{Fluxo Óptico}

Fluxo Óptico é a distribuição 2D da velocidade de cada pixel associado a um vetor de velocidade, em que a utilização da diferença entre tons de cinza em duas imagens é possível saber se houve movimento (BARBOSA et al., 2005) (MOTA; 2012). Segundo Oliveira-Neto (2014) Fluxo Óptico é definido como uma técnica para estimar movimento através de intensidades com os mesmos padrões, podendo assim identificar objetos em outra imagem. Existem várias outras aplicações para fluxo óptico além de estimar movimento, tais como visão robótica, registro de imagens médicas e codificação de faces.

\subsection{Métodos de Fluxo Óptico}

Os métodos de Fluxo Óptico são classificados por técnicas diferenciais, técnicas baseadas em frequência/energia e técnica por correlação. A técnica diferencial calcula em uma imagem 2D o deslocamento de uma situação real, através de derivadas obtidas pelo espaço percorrido em um curto tempo considerando que a intensidade do brilho em outros frames seja constante (LARA, 2006).

Oliveira (2003) afirma que quando uma cena filmada por uma câmera, e essa cena tem algum tipo de deslocamento, ocorre uma mudança nas imagens capturadas (frames) em relação aos pixels. As características desses pixels são armazenadas em um vetor de velocidade denominado de campo de deslocamento

$$
\text { A técnica baseada em }
$$
frequência/energia utiliza a transformada de Fourier que transforma as imagens do domínio espacial para o domínio da frequência. Uma grande vantagem dessa técnica é que as alterações em relação à luminância não interferem nos cálculos realizados para estimar do Fluxo Óptico (LARA, 2006).

A técnica por correlação procura características peculiares de uma imagem 
com a imagem seguinte. Os cantos e contornos encontrados em uma imagem são bons exemplos de características a terem correlação. Esta técnica é utilizada quando se tem grande taxa de ruídos ou poucos frames em uma cena (LARA, 2006).

\subsection{Erros de Definição do Fluxo Óptico}

Segundo Minetto (2007) existem três situações que podem acarretar uma definição errada do Fluxo Óptico: problema de oclusão, ambiguidade na correspondência óptica e incoerência espacial.

O problema de oclusão é quando um ponto identificado em uma imagem não é encontrado na imagem seguinte. Por exemplo, um ponto $P$ em um frame $/$ é visível e não aparece no frame $1+1$, como mostra a Figura 1.

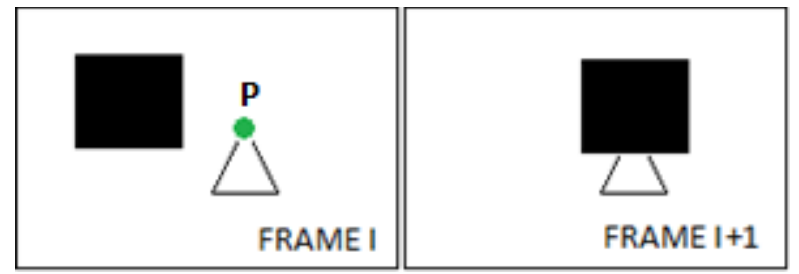

Figura 1. Problema de oclusão de pontos. Fonte: (MINETTO, 2007).

A ambiguidade na correspondência óptica, também conhecida como problema de abertura, é quando um mesmo ponto tem ambiguidades no frame seguinte. Isto quer dizer que um ponto a no frame I pode aparecer vários pontos no frame I+1. Essa ambiguidade pode ser unidimensional (pontos encontrados estão na mesma linha) ou bidimensional (pontos encontrados em linhas diferentes). A Figura 2 ilustra esse problema de ambiguidade.

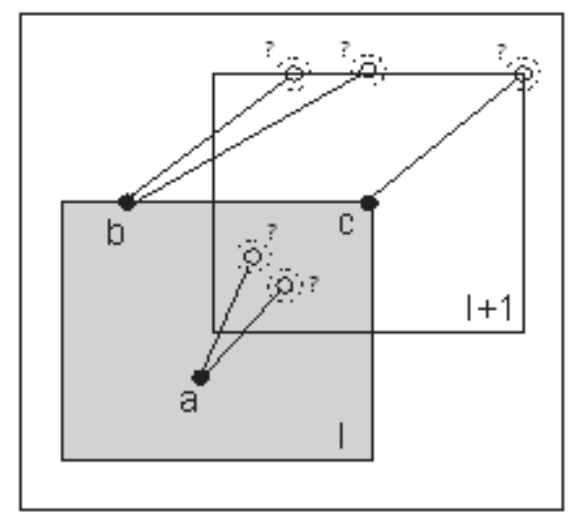

Figura 2. Problema de ambiguidade dos pontos.

Fonte: (MINETTO, 2007).

Incoerência espacial é quando pontos distintos de um mesmo objeto seguem um fluxo incomum em relação ao deslocamento do objeto. Por exemplo, um objeto com deslocamento para a direita possuindo alguns pontos com fluxo diferente (Figura 3).

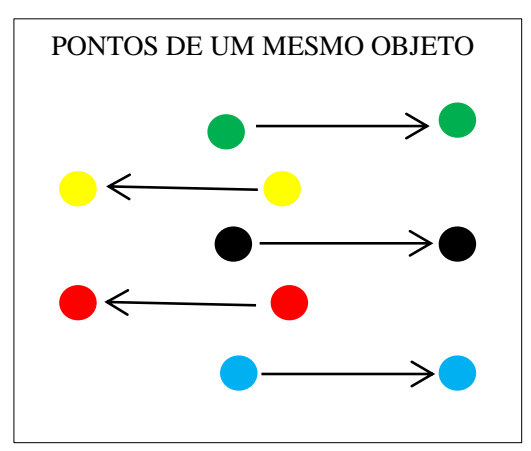

Figura 3. Problema de incoerência espacial dos pontos.

Fonte: (MINETTO, 2007).

\subsection{Algoritmo de Lucas e Kanade}

Lucas e Kanade (1981) descrevem o método como uma técnica em que uma imagem (frame) é dividida em partes, 
calculando o Fluxo Óptico para cada uma

$$
F(h+x)=G(x)
$$

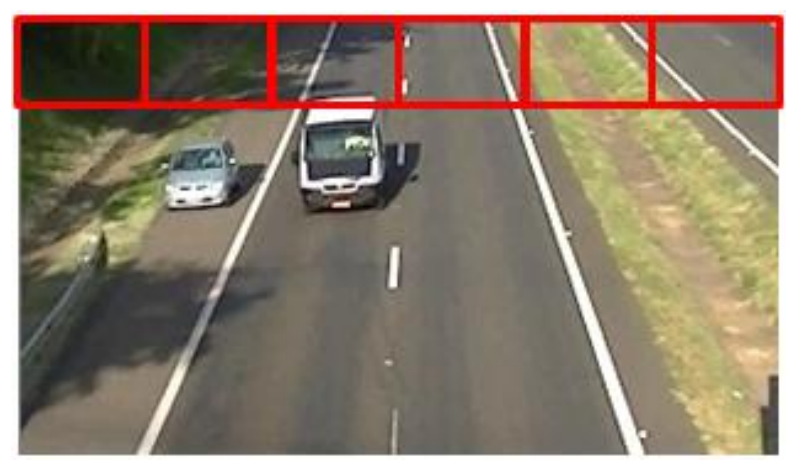

Figura 4. Divisão do frame em $N$ partes para aplicação do algoritmo de Lucas e Kanade (1981).

A partir das definições do algoritmo de Lucas e Kanade (1981) assume-se a hipótese em um vídeo analisado, que pixels diferentes em frames diferentes representam o mesmo ponto de um objeto que esteja em movimento, possuindo intensidades equivalentes, isto é, possuem brilho, contraste e cor com pouca mudança. Em geral, a taxa de captura de câmeras de vídeo (frames por segundo) é maior que a velocidade dos objetos, sendo assim 0 movimento dos objetos é relativamente pequeno de um frame para outro (BRITO, 2013).

Em uma região dentro de uma imagem descrita por uma função $F(x)$, que representa o valor do pixel, dada sua posição, se em uma segunda imagem, a mesma região é representada por outra função $G(x)$, então existe um valor $h$ tal que (LUCAS; KANADE, 1981):
Devido a possibilidade de existir ruídos nas imagens, faz-se necessário estimar o erro $(\varepsilon)$ e a Equação a seguir (2) apresenta uma alternativa.

$$
\varepsilon=\sum_{X \in \mathbb{R}}|F(h+x)-G(x)|^{2}
$$

A equação final para o cálculo do Fluxo Óptico de Lucas e Kanade é definida como:

$$
\mathrm{h}=\left[\sum_{\mathrm{R}}\left(\frac{\partial \mathrm{F}(\mathrm{x})}{\partial \mathrm{x}}\right)^{\mathrm{T}} \cdot[\mathrm{G}(\mathrm{x})-\mathrm{F}(\mathrm{x})]\right] \cdot\left[\sum_{\mathrm{R}}\left(\frac{\partial \mathrm{F}(\mathrm{x})}{\partial \mathrm{x}}\right)^{\mathrm{T}} \cdot\left(\frac{\partial \mathrm{F}(\mathrm{x})}{\partial \mathrm{x}}\right)\right]^{-1}
$$

\section{5 - Algoritmo de Kanade, Lucas e Tomasi} (KLT)

O algoritmo com bom desempenho é aquele capaz de identicar os pontos com grandes diferenças de deslocamento de um frame para outro. O algoritmo KLT utiliza pirâmides na implementação do algoritmo analisando imagens menores do mesmo frame e selecionando os pontos com melhores características (ROCHA, 2012).

$$
\text { Minetto (2007) afirma que o }
$$
algoritmo KLT calcula o Fluxo Óptico em múltiplas escalas de resolução, começando com a resolução mais baixa e chegando até a resolução original. A coleção de imagens obtidas é chamada de pirâmide e é ilustrada na Figura 5. 


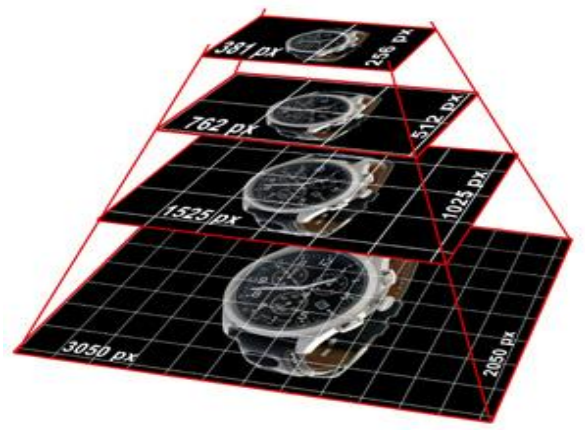

Figura 5. Pirâmide do Algoritmo KLT.

De acordo com Rocha (2012) uma mesma cena fornece um conjunto de imagens que são calculadas recursivamente. Partindo de uma imagem inicial I no nível mais alto da piramide $(L m)$, de modo que o próximo nível é calculado a partir do nível anterior e assim sucessivamente, ou seja, o Fluxo Óptico é calculado no nível $L m$ da pirâmide obtendo o vetor de Fluxo Óptico $v^{\rightarrow L}=\left[u^{L} v^{L}\right]^{T}$, onde $u$ é vetor de velocidade em $x$, e $v$ é o vetor de velocidade em $y$. Esses vetores são utilizados como valor inicial no nível $L m-1$, os vetores obtidos nesse nível são utilizados no nível $L m-2$, e assim sucessivamente. Para o nível inicial $\operatorname{Lm}$ o vetores $u$ e $v$ são nulos (Figura 6).

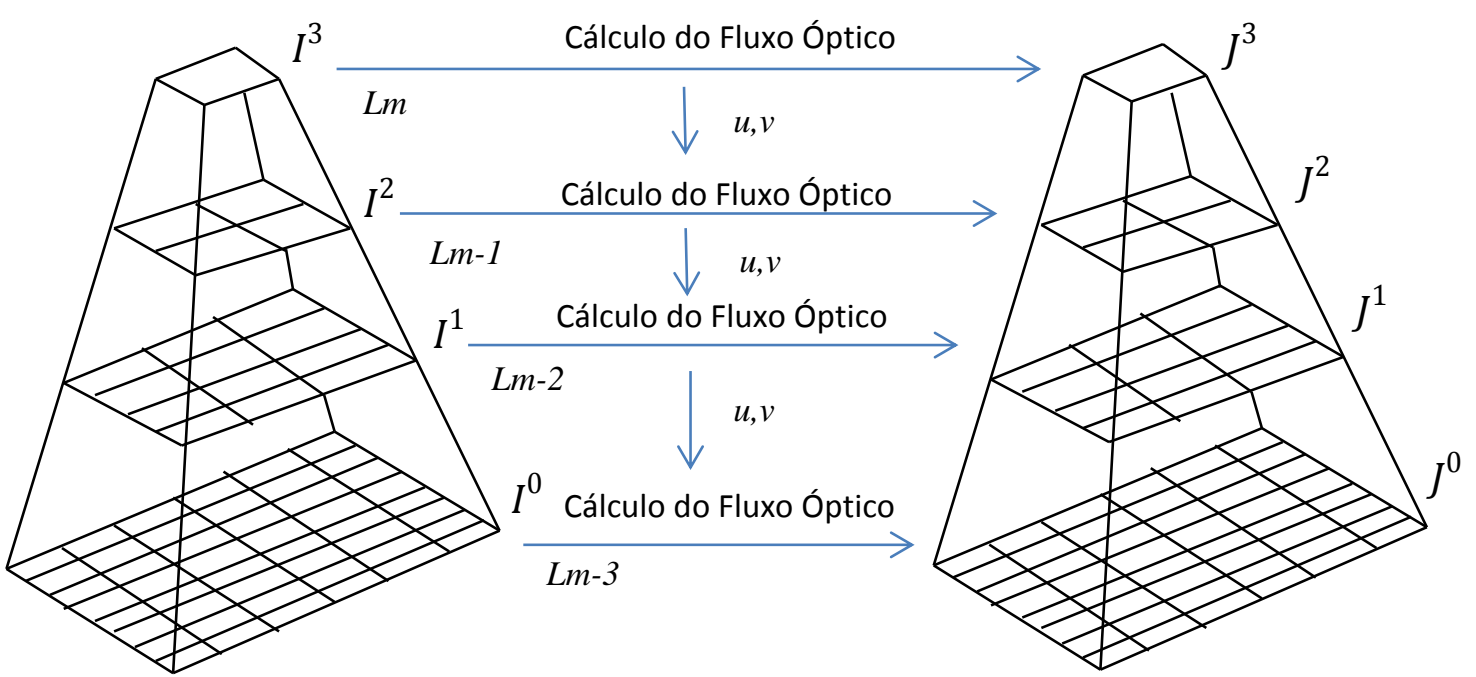

Figura 6. Utilização dos vetores $u, v$ no algoritmo KLT.

\subsection{Good Features to Track}

Uma boa escolha dos pontos a serem analisados faz com que o algoritmo tenha maior desempenho e menor custo computacional, geralmente esses pontos são os cantos e arestas de uma imagem (MACEDO, 2013).
Good Features to Track é baseado no algoritmo de Shi Tomasi (1994) e segundo Dihl (2009) utiliza seleção de características pontuais, o rastreamento desses pontos é feito pela similaridade em uma pequena região, tanto no frame atual quanto no frame 
seguinte. O KLT pode detectar os pontos de uma maneira mais rápida, desde que o deslocamento dos pontos analisados não seja grande de um frame para outro. A Figura 7 mostra um exemplo de aplicação do algoritmo Shi Tomasi (1994).

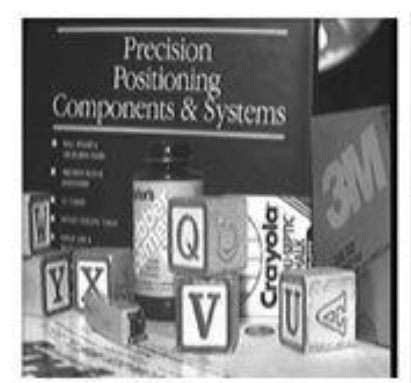

(a)

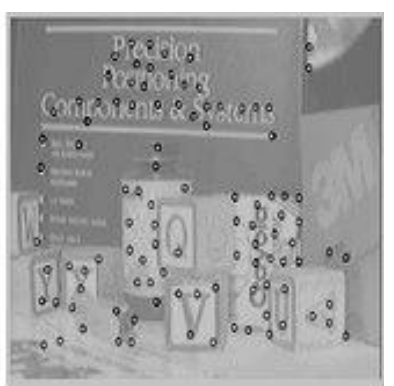

(b)
Figura 7. (a) - Imagen inicial (b) - Pontos encontrados levando em consideração a textura com o metodo de Shi Tomasi (1994).

Fonte: (DIHL, 2009).

\section{METODOLOGIA DESENVOLVIDA}

O Fluxograma da Metodologia desenvolvida neste trabalho para identificação e rastreamento de veículos pode ser visualizado na Figura 8.

Os frames são obtidos do vídeo em pares para aplicar a técnica de Fluxo Óptico. Após a obtenção dos frames é realizada a etapa de pré-processamento, onde os frames são redimensionados a uma razão de 1:4, por exemplo, um frame com resolução de $1280 \mathrm{x}$ 1024 pixels é redimensionado para uma resolução de $320 \times 256$ pixels. Ainda na etapa de pré-processamento, os frames são convertidos em escala de cinza.

Em seguida para identificar os melhores pontos a serem rastreados foi utilizado o algoritmo de Shi e Tomasi (1994), que segundo Sobral (2012) é um extrator de características que seleciona os melhores pontos (pixels) a serem rastreados em um frame do vídeo.

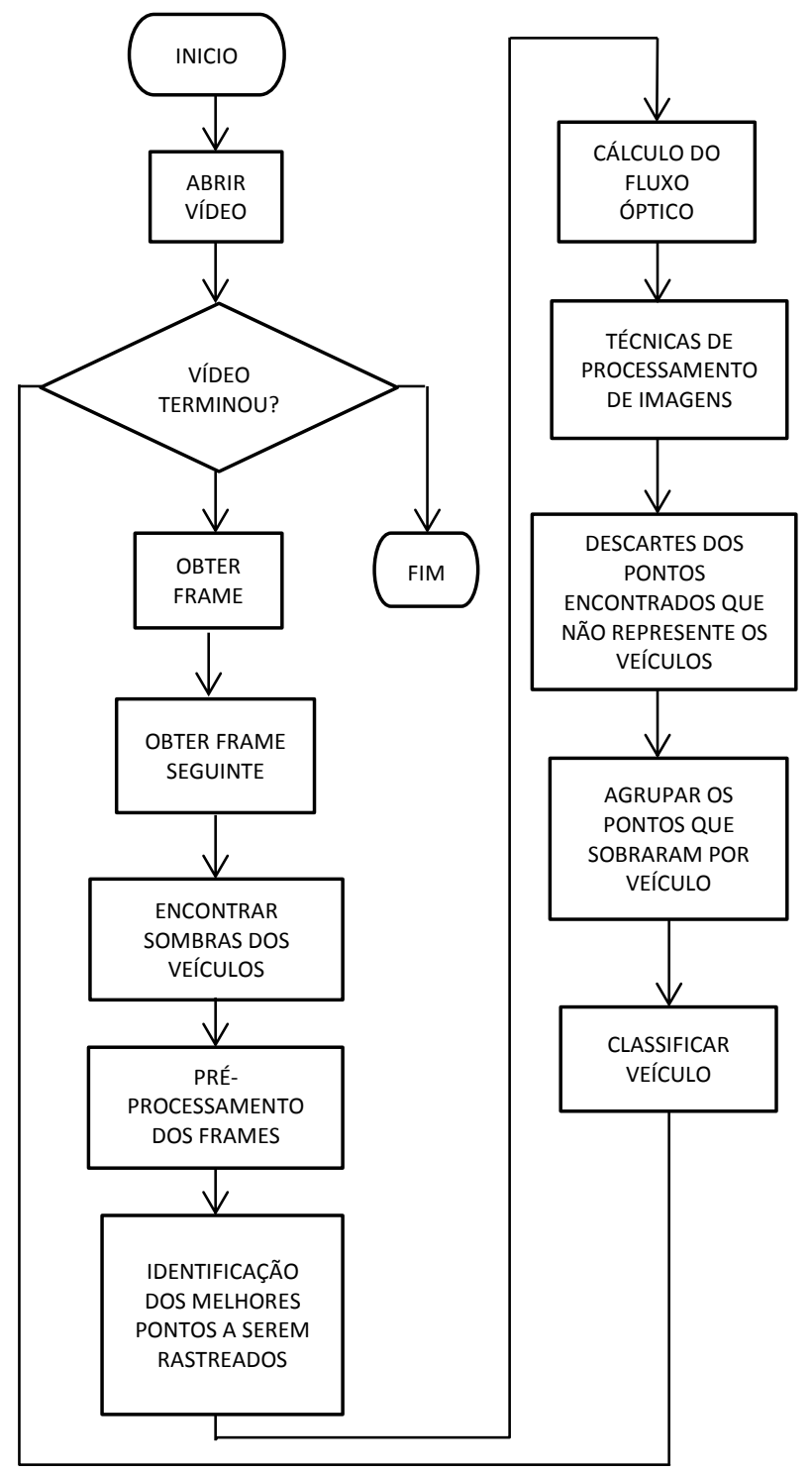

Figura 8. Fluxograma do algoritmo referente à Metodologia Desenvolvida.

A técnica de Shi e Tomasi (1994) é utilizada no primeiro frame de cada par obtido no processo, se repetindo até o vídeo terminar. Na Figura 9 têm-se os melhores pontos a serem rastreados por meio da aplicação deste algoritmo. 


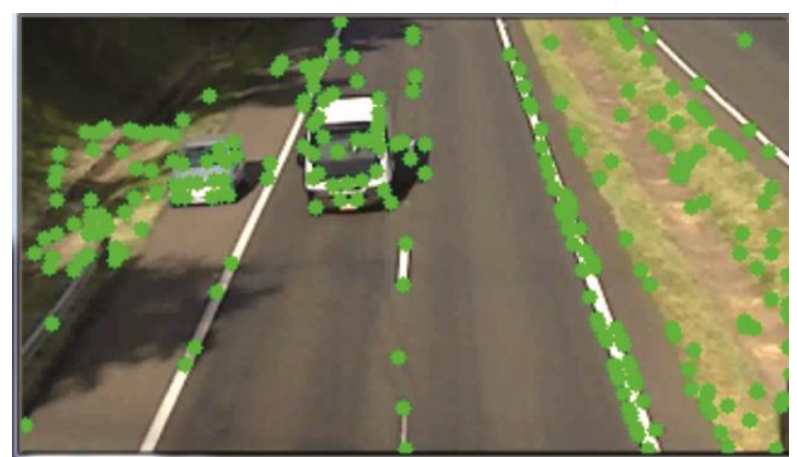

Figura 9. Pontos rastreados pelo algoritmo de Shi e Tomasi (1994).

Após encontrar os melhores pontos a serem rastreados é realizado o cálculo do Fluxo Óptico, que tem a finalidade de encontrar em outro frame os pontos correspondentes identificados na etapa anterior. Para calcular o Fluxo Óptico foi empregado o algoritmo de Lucas e Kanade (1981) por meio da biblioteca de Visão Computacional OpenCV. A Figura 10 mostra o deslocamento dos pontos, de maneira que o início da seta representa os pontos encontrados no primeiro frame e o final da seta os pontos encontrados pelo algoritmo de Fluxo Óptico.

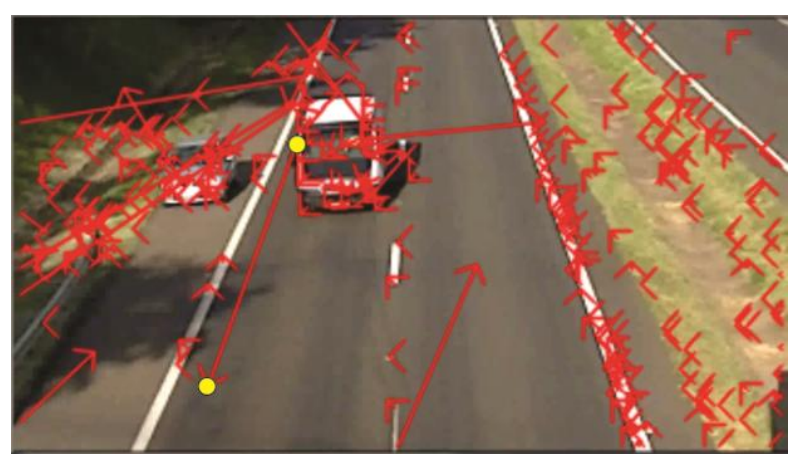

Figura 10. Deslocamento de um ponto de um frame para outro.

A etapa seguinte consiste em encontrar as sombras que pertencem aos veículos, para que no momento de calcular a área dos veículos encontrados, as sombras não sejam consideradas como parte dos veículos. Para eliminar as sombras, o algoritmo primeiramente encontra a imagem de fundo (background) e considera o frame atual como foreground. Ambos são convertidos no espaço de cor HSV (Hue, Saturation e Value), como ilustram as Figuras 11 e 12.

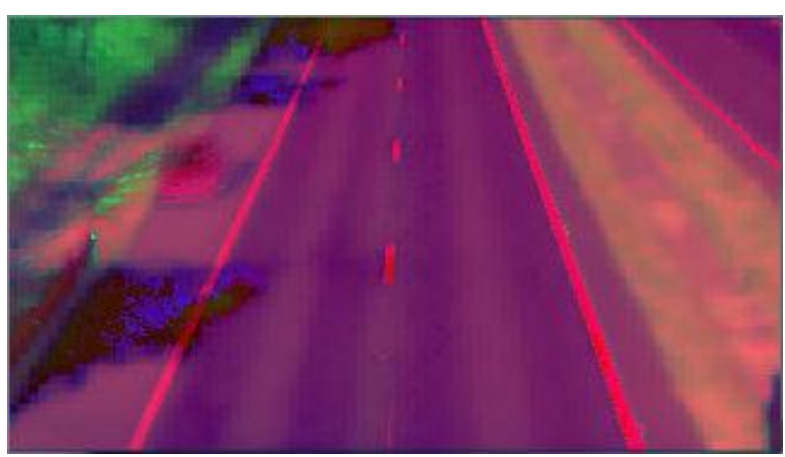

Figura 11. Background no espaço de cor HSV.

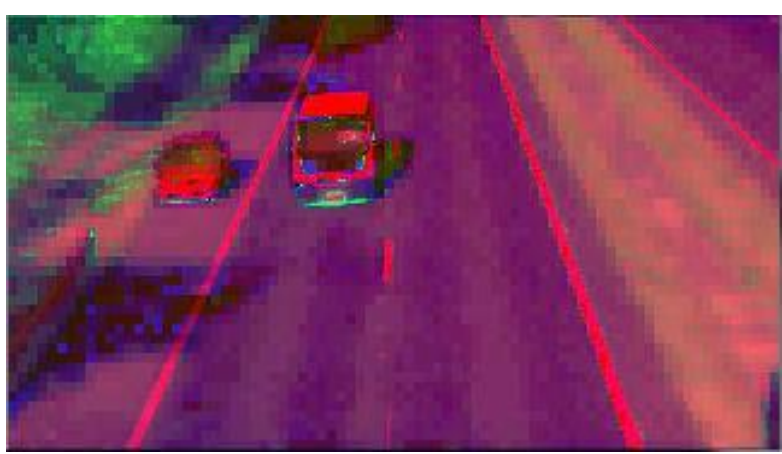

Figura 12. Foreground no espaço de cor HSV.

Os canais HSV das imagens foreground como para o background foram separados em três. Apenas são utilizados os canais referentes a luminosidade (Value), sendo os outros canais descartados. Com a imagem resultado da divisão do canal Value apresentada na Figura 13 é realizada a normalização em cada posição da imagem, e os valores encontrados entre 0.2 e 0.9 são 
considerados como sombra, já valores fora dessa faixa são desconsiderados. A Figura 14 mostra a sombra encontrada.

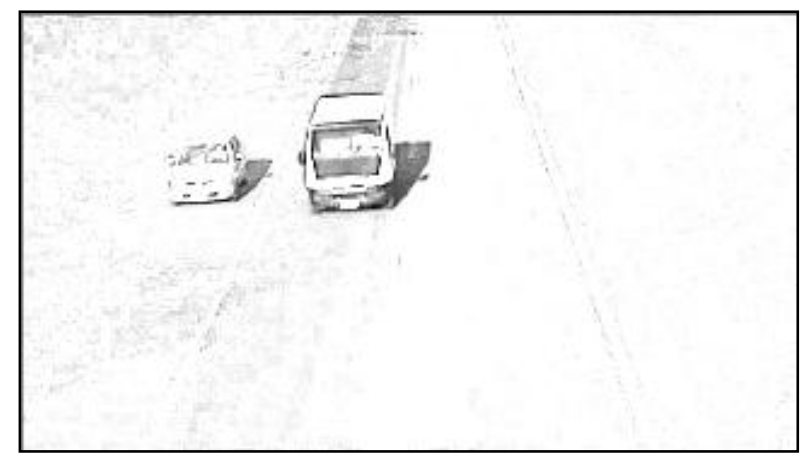

Figura 13. Imagem Resultado da divisão do canal Value das duas imagens.

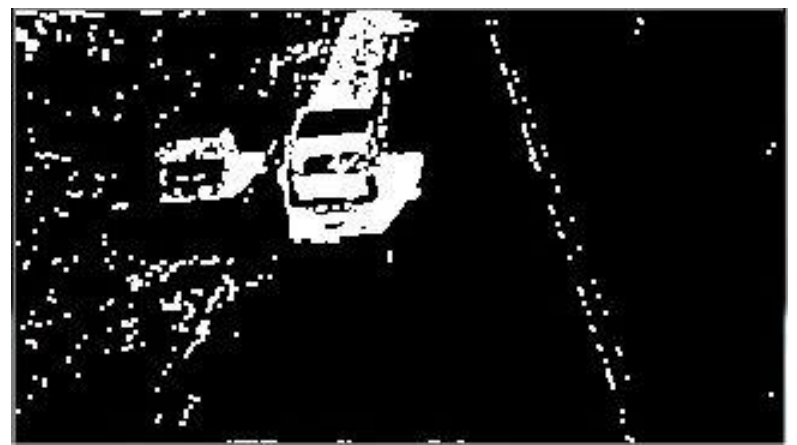

Figura 14. Sombra do veículo detectado.

A próxima etapa é utilizar técnicas de processamento de imagens para eliminar as sombras dos veículos encontrados. $\mathrm{O}$ primeiro passo nessa etapa é realizar a subtração dos dois frames obtidos de cada par de frames. Após essa etapa, conclui-se o pré-processamento. As Figura 15 e 16 mostram um exemplo de um par de frames, enquanto que na Figura 17 é mostrado o resultado da subtração desses dois frames.

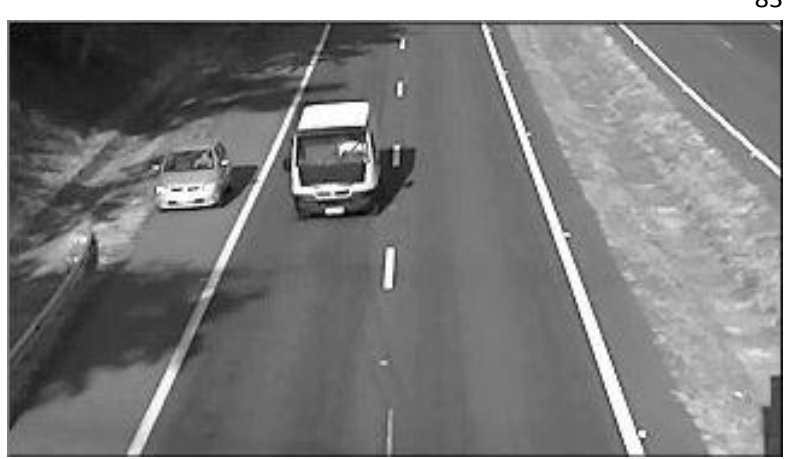

Figura 15. Primeiro frame obtido de um par.

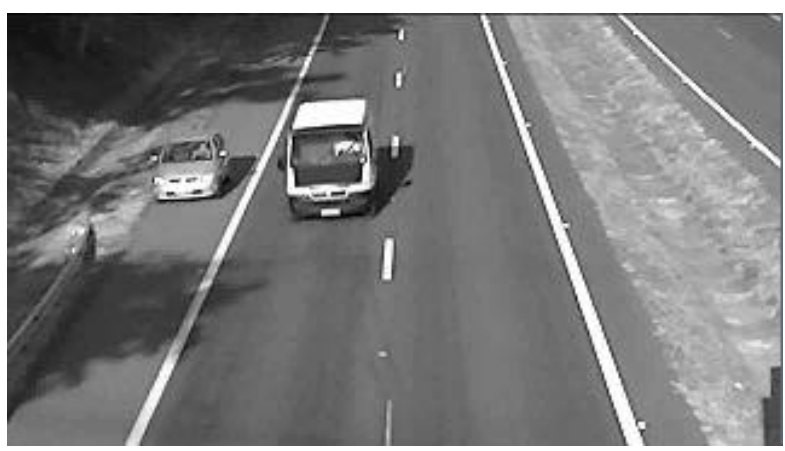

Figura 16. Segundo frame obtido de um par.

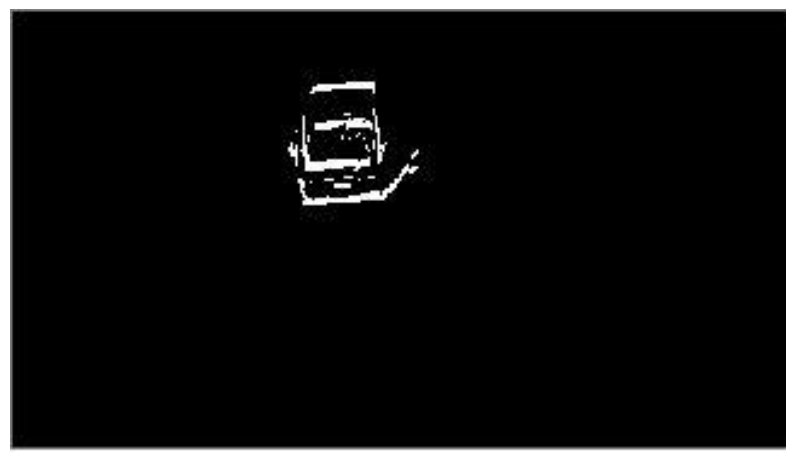

Figura 17. Resultado da subtração dos dois frames de um par.

O passo seguinte nessa etapa é encontrar uma imagem que represente o veículo sem a sombra (Figura 18), sendo ela utilizada na etapa de classificação dos veículos. Para isso, foi realizada a subtração da imagem que representa as sombras do veículo (Figura 14) com a que representa o veículo, com sua sombra (Figura 17). O resultado da subtração das imagens é 
apresentado na Figura 18, já a Figura 19 mostra o resultado da operação de dilatação da morfologia matemática aplicada no resultado da subtração das imagens. Nessa operação de dilatação foi usado um elemento estruturante $15 \times 15$ na forma de um quadrado com o ponto de origem na posição central da imagem.

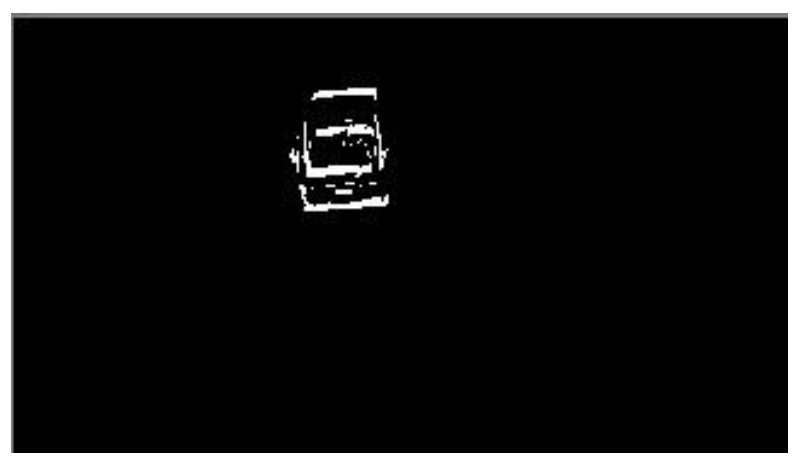

Figura 18. Resultado da subtração das imagens que correspondem a sombra (Figura 14) com a imagem resultado da subtração dos dois frames de um par (Figura 17).

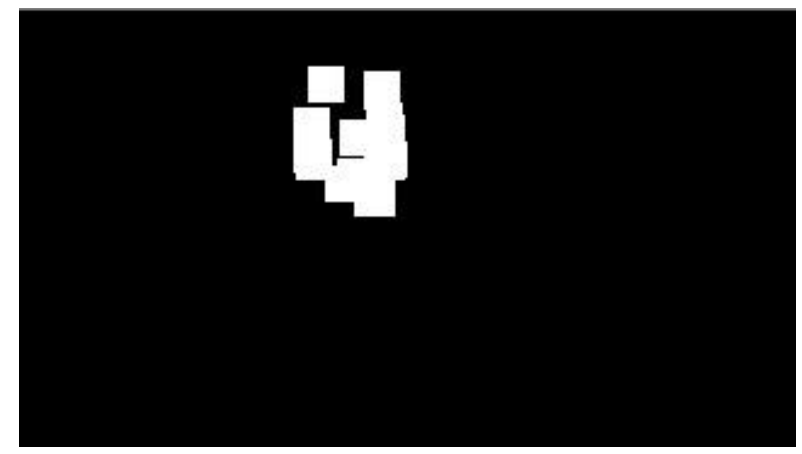

Figura 19. Resultado das aplicação da operação de dilatação na imagem da Figura 18.

Em seguida foi realizado o descarte dos pontos encontrados que não representam os veículos. Para isso, foram implementadas duas funções, uma que elimina os pontos encontrados por onde os veículos não trafegam, como os canteiros da rodovia, e outra que verifica a coerência dos deslocamentos dos pontos restantes. Nesse processo, o maior problema foi manter apenas os pontos encontrados que estão somente na rodovia. Isto porque, em cada linha que o carro se movimenta na imagem, a coluna que representa a margem da rodovia também se altera, isso porque a margem da rodovia não está em um ângulo de 90 graus. A solução encontrada para resolver esse problema foi aplicar a cálculo de intersecção das retas (Equações 4, 5 e 6), nas duas margens da rodovia, e os pontos de interesse que estiverem entre as margens são considerados, como mostrado na Figura 20.

$$
\begin{array}{r}
P 1 x=\frac{P 1 y-b}{a} \\
b=P 2 y-(a \cdot P 2 x) \\
a=\frac{P 2 y-P 3 y}{P 2 x-P 3 x}
\end{array}
$$

onde $b$ é coeficiente linear e $a$ é o coeficiente angular.

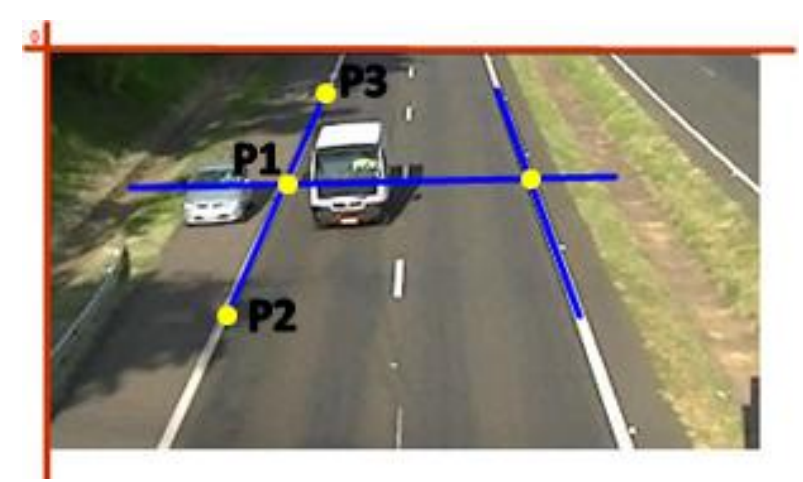

Figura 20. Delimitação das margens da rodovia.

Para eliminar os pontos incoerentes, foi analisada a direção de deslocamento dos pontos, ou seja, os pontos com direções 
diferentes da direção que o veículo trafega são eliminados. A Figura 21 mostra o resultado depois de aplicado as duas funções (descarte dos pontos que não estão na rodovia e incoerência da direção dos deslocamento dos pontos).

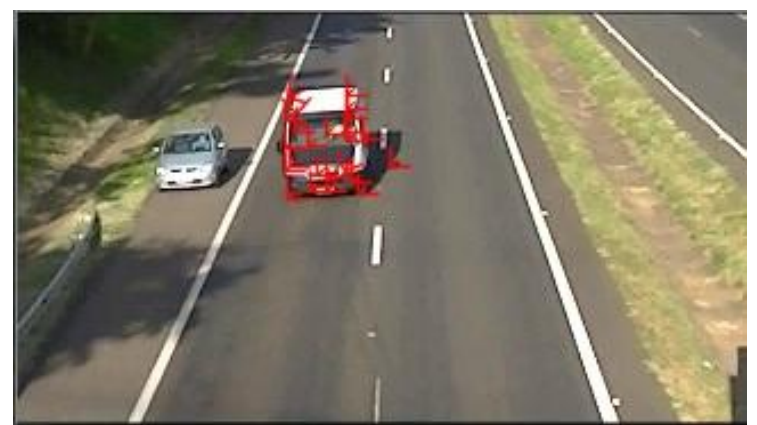

Figura 21. Resultado depois de eliminar os pontos sem interesse.

Para classificar os veículos, primeiramente foram agrupados os pontos que estavam próximos, utilizando a distância Euclidiana (Equação 7). Cada conjunto de pontos restante representa um veículo, em que foi estabelecido um retângulo mínimo ao redor desse conjunto. Porém, tentou-se a abordagem baseada no cálculo da Distância Euclidiana, mas esta se mostrou ineficiente por não enquadrar completamente o veículo, conforme ilustrado na Figura 22.

$$
\text { dist }=\sqrt{(x 1-x 2)^{2}+(y 1-y 2)^{2}}
$$

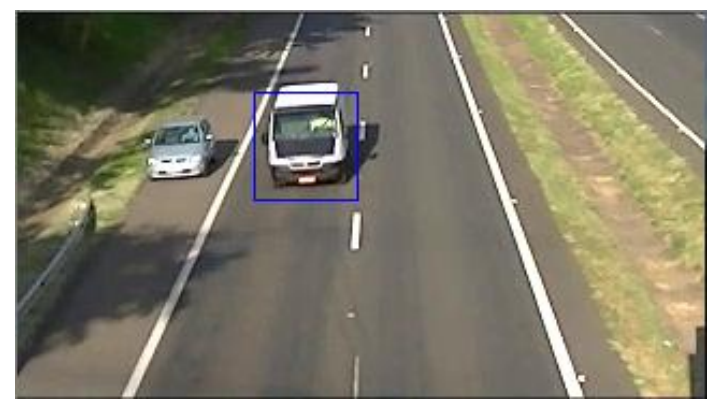

Figura 22. Enquadramento incorreto do veículo.
Para que o retângulo enquadre correntemente o veículo, foi utilizada a imagem resultante da operação de dilatação, representada pela Figura 19, em que os lados do retângulo foram redimensionados a fim de enquadrar toda a parte branca, que representa o veículo (Figura 23).

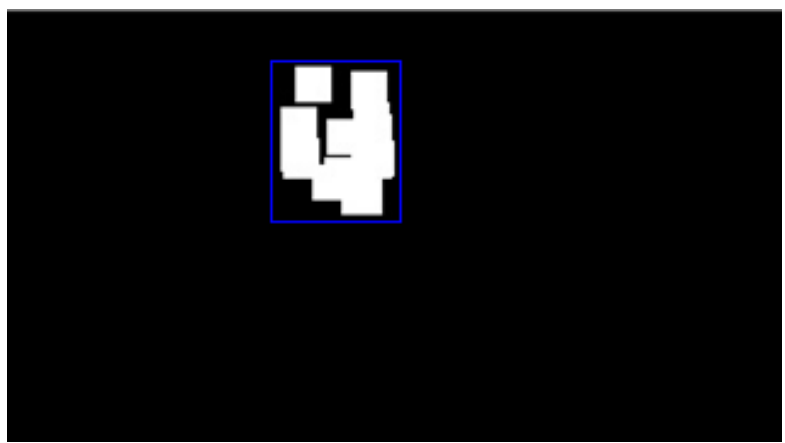

Figura 23. Resultado do enquadramento do veículo.

$$
\text { Com o retângulo enquadrado }
$$
corretamente sobre o veículo foi calculada a área do retângulo, classificando os veículos como: pequeno, médio e grande porte. O cálculo da área é realizado somente quando o lado inferior do retângulo tocar a linha vermelha (Figura 24), que foi estabelecida empiricamente. É nessa região da imagem que foi constatado um melhor número de acertos na classificação dos veículos.

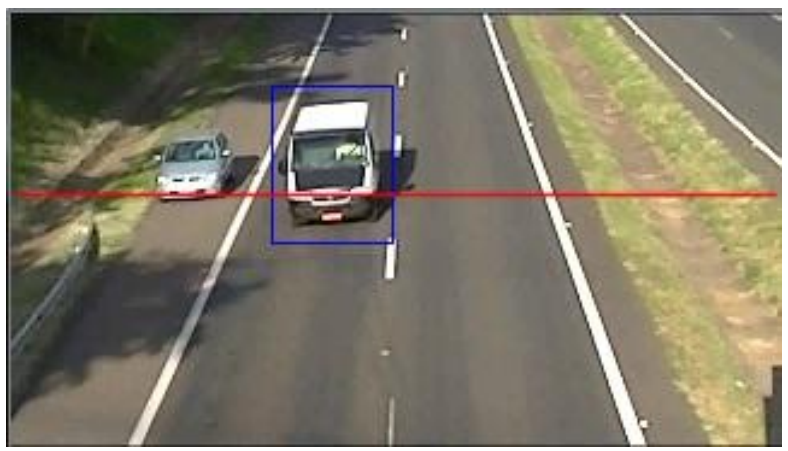

Figura 24. Classificação do veículo. 


\section{EXPERIMENTOS}

Foi realizada a gravação de um vídeo na rodovia Raposo Tavares na cidade de Presidente Prudente/SP durante 12 minutos e 22 segundos. O vídeo foi gravado com resolução de 1920 x 1080 pixels com 30 frames por segundo, sendo esse vídeo filmado a favor do sol, para que a qualidade do vídeo fosse a melhor possível. Para realizar os testes, apenas foi utilizada uma região do vídeo (1280 x 720 pixels) para melhor visualização dos veículos. Também foi reduzido o tempo de duração do vídeo para 5 minutos, pois existiam momentos no vídeo em que não constava tráfego de veículos.

Para realizar os Experimentos foi utilizado um computador com processador i5-2467M, 4 GB de memória RAM e placa de vídeo integrada Intel HD Graphics 3000. O tempo total de processamento de cada etapa do processo mostrado na Figura 8 é de aproximadamente 210 milisegundos.

\section{RESULTADOS}

No vídeo de 5 minutos utilizado, trafegaram pela rodovia 45 veículos envolvendo pequeno, médio e grande porte. Um veículo de médio porte não foi identificado, e um veículo foi classificado como de grande porte, ao invés de veículo de médio porte. A Tabela 2 mostra os resultados do algoritmo.
Tabela 2. Resultados dos experimentos realizados.

\begin{tabular}{l|c|c|c}
\hline & Total & Acertos & Erros \\
\hline $\begin{array}{l}\text { Veículo } \\
\text { de Pequeno Porte }\end{array}$ & 7 & 7 & 0 \\
\hline $\begin{array}{l}\text { Veículo de Médio } \\
\text { Porte }\end{array}$ & 28 & 27 & 1 \\
\hline $\begin{array}{l}\text { Veículo de Grande } \\
\text { Porte }\end{array}$ & 10 & 9 & 1 \\
\hline Total & 45 & 43 & 2 \\
\hline \multicolumn{1}{c}{ O veículo de médio porte não foi }
\end{tabular}

classificado porque o mesmo estava no acostamento e quando o veículo ultrapassou a região onde são realizadas as classificações (linha vermelha da Figura 26), ele se encontrava em baixa velocidade, e os pontos encontrados no veículo para realizar o Fluxo Óptico não foram suficientes para classificalo, como mostra a Figura 25.

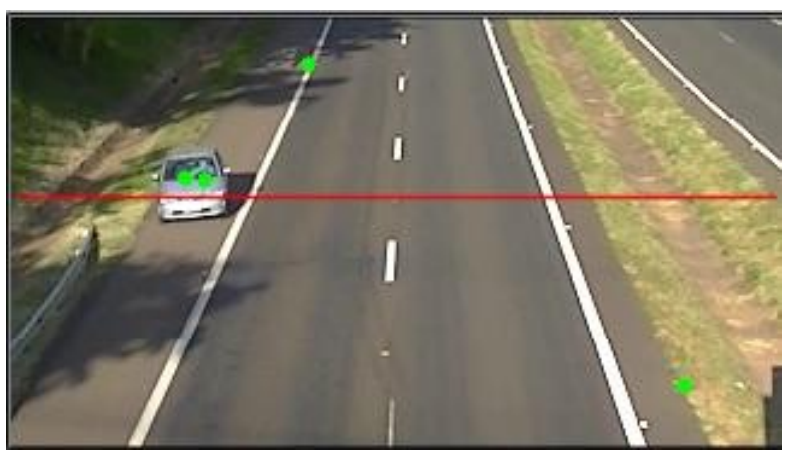

Figura 25. Veículo não classificado.

O veículo que foi classificado incorretamente, trafegava muito próximo a outro no momento da classificação do veículo, em que um veículo de médio porte foi classificado como veículo de grande porte, como pode ser visto na Figura 26. 


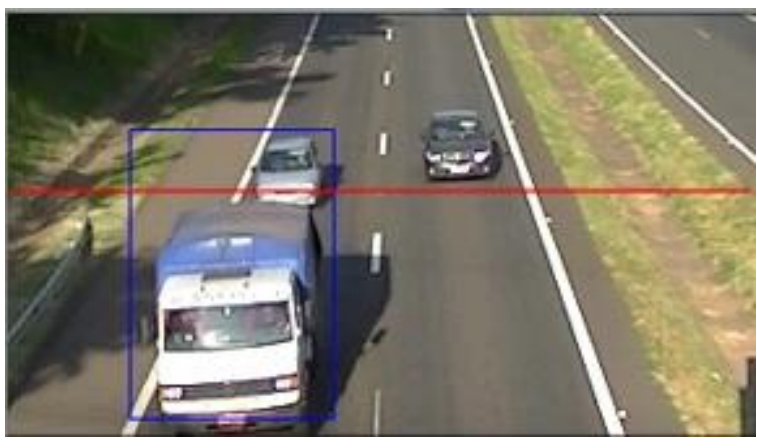

Figura 26. Veículo de médio porte classificado como grande porte.

\section{CONSIDERAÇÕES FINAIS}

O trabalho realizado apresentou bons resultados, como pode ser visto na Tabela 2, além de ter um tempo de processamento relativamente baixo. Porém, neste trabalho não foi tratado o problema de oclusão de veículos que estavam muito próximos. Acredita-se que a altura em que a câmera foi colocada no momento da filmagem não estava adequada, e os problemas causados pelas oclusões de alguns veículos poderiam ser reduzidos se a câmera estivesse em um ponto mais alto.

A velocidade média dos veículos que trafegaram pela rodovia, durante a filmagem, foi de $90 \mathrm{~km} / \mathrm{h}$, não sendo possível realizar experimentos com veículos em alta velocidade, já que a via não permitia.

Acredita-se que, com o uso de um computador com um poder de processamento maior, e ainda com uma placa de vídeo dedicada, poderia melhorar o desempenho da metodologia do trabalho desenvolvido. Vale também considerar, em um trabalho posterior, o uso de algoritmos que são processados em placas de vídeo com CUDA, e com isso enriquecer em termos de desempenho e se obter processamento em tempo real.

\section{REFERÊNCIAS}

BARBOSA, R.L. et al. A computação do Fluxo Óptico em imagens obtidas por um sistema móvel de mapeamento terrestre. Revista da Sociedade Brasileira de Cartografia, Geodésia, Fotogrametria e Sensoriamento Remoto - SBC, v.57/2, p.72-78, 2005.

BRITO, G.R. Desenvolvimento de algoritmo para tracking de veículos. 2011. 45f. Trabalho de conclusão de curso (Bacharel) Escola Politécnica da universidade de São Paulo, Departamento de Engenharia Elétrica. São Paulo - SP.

CHEN, T.; LIN, Y.; CHEN, T. Intelligent vehicle counting method based on blob analysis in traffic surveillance. In: SECOND INTERNATIONAL CONFERENCE ON INNOVATIVE COMPUTING, INFORMATION AND CONTROL, ICICIC'07. Proceedings... p.238-242, 2007.

DIHL, L.L. Ratreamento de objetos usando descritores estatísticos. 2009. 69f. Dissertação (Mestrado). Universidade do Vale do Rio dos Sinos, São Leopoldo - RJ.

LARA, A.C. Segmentação de movimento para aplicações de vigilância eletrônica. 2006. 38f. Dissertação (Mestrado) - Departamento de Ciências da Computação e Instituto de Matemática e Estatística da Universidade de São Paulo, São Paulo - SP.

LUCAS, B.D.; KANADE, T. An Iterative Image Registration Technique with an Application to Stereo Vision. In: PROCEEDINGS OF THE 7TH INTERNATIONAL JOINT CONFERENCE ONARTIFICIAL INTELLIGENCE (IJCAI '81). Proceedings... 1981, p.674-679. 
MACEDO, S.V.M. Aplicação do Teste Kens para detecção de outliers em fluxo óptico. 2013. 51f. Dissertação (Mestrado) Universidade Federal de Pernambuco. Fortaleza-CE.

MINETTO, R. Detecção robusta de movimento de câmera em vídeos por análise de fluxo óptico ponderado. 2007. 61f. Dissertação (Mestrado) - Universidade Estadual de Campinas, Campinas - SP.

MOTA, V.F. Tensor baseado em fluxo ótico para descrição global de movimentos em vídeos. 2011. 179f. Dissertação apresentada ao programa de pós-graduação para obtenção de grau mestre - Universidade Federal de Juiz de Fora. Juiz de Fora - MG.

OLIVEIRA-NETO, V.J.; GOMES, D.M. Comparação de métodos para localização de Fluxo Óptico em sequências de imagens. In: SIBGRAPI CONFERENCE ON GRAPHICS, PATTERNS AND IMAGES (SIBGRAPI), 27. Proceedings... 2014. p.298-303,

OLIVEIRA, I.N.S. Metodologia para detecção rápida de movimento em sequências de imagens. 2003. 180f. Dissertação (Doutorado) - Instituto de Física de São Carlos da Universidade de São Paulo, São Carlos SP. http://dx.doi.org/10.11606/t.76.2003.tde$\underline{05052008-173600}$

ROCHA, T. Uma proposta para classificação de ações humanas baseada nas características do movimento em redes neurais artificiais. 2012. 83f. Dissertação (Mestrado) - Universidade Federal de Brasília, Brasília - DF.

SOBRAL, A.C. Classificação automática do estado do trânsito baseada em contexto global. 2012. 94 f. Dissertação (Mestrado) Universidade Federal da Bahia, Escola Politécnica, Salvador - BA.
SHI, J.; TOMASI, C. Good features to track. In: IEEE COMPUTER SOCIETY CONFERENCE ON COMPUTER VISION AND PATTERN RECOGNITION (CVPR'94). Proceedings... 1994. p. 593-600.

TANNÚS, H.C; SOARES, V.C.V; COSTA, R.M. Técnicas de processamento digital de imagens aplicadas na contagem volumétrica de veículos. In: VIII WORKSHOP DE VISÃO COMPUTACIONAL (WVC 2012). Anais... Goiás: UFG, 2012. 